\title{
Monitoreo de la superficie de los bosques nativos de Chile: un desafío pendiente
}

\author{
Monitoring Chilean native forest area: a pending challenge
}

\author{
Alejandro Miranda a,b,d*, Antonio Lara ${ }^{\mathrm{c}, \mathrm{d}}$, Adison Altamirano a , Carlos Zamorano-Elgueta ${ }^{\mathrm{i}, \mathrm{d}}$, \\ H Jaime Hernández ${ }^{\mathrm{e}}$, Mauro E González ${ }^{\text {,dd }}$, Aníbal Pauchard ${ }^{\mathrm{f}, \mathrm{g}}$, Álvaro Promis ${ }^{\mathrm{h}}$ \\ *Autor de correspondencia: ${ }^{a}$ Universidad de La Frontera, Departamento de Ciencias Forestales, \\ Laboratorio de Ecología del Paisaje Forestal, P.O. Box 54-D, Temuco, Chile, alejandro.miranda@ufrontera.cl \\ ${ }^{\mathrm{b}}$ Universidad Austral de Chile, Facultad de Ciencias Forestales y Recursos Naturales, Escuela de Graduados, Valdivia, Chile. \\ c Universidad Austral de Chile, Facultad de Ciencias Forestales y Recursos Naturales, Instituto de Conservación, \\ Biodiversidad y Territorio, Valdivia, Chile. \\ d Universidad de Chile, Center for Climate and Resilience Research (CR2), Santiago, Chile. \\ e Universidad de Chile, Facultad de Ciencias Forestales y de la Conservación de la Naturaleza, \\ Laboratorio de Geomática y Ecología del Paisaje. Santiago, Chile. \\ ${ }^{\mathrm{f}}$ Universidad de Concepción, Facultad de Ciencias Forestales, Laboratorio de Invasiones Biológicas, \\ Victoria 631, Casilla 160-C,Concepción, Chile. \\ g Instituto de Ecología y Biodiversidad (IEB), Las Palmeras 3425 Ñuñoa, Casilla 653, Santiago, Chile.

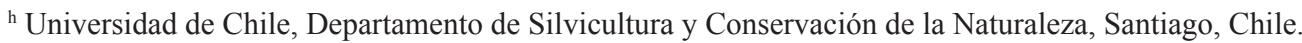 \\ ${ }^{\mathrm{i}}$ Universidad de Aysen. Obispo Vielmo 62, Coyhaique, Chile.
}

\section{SUMMARY}

Forest monitoring is important for decision making of forest management and conservation. In Chile, the forest monitoring system was initiated in 1994, which has been continued through the implementation of regional updates. This has enabled a temporal monitoring of the distribution and surface covered by native forest. However, while regional updates have reported increase in the surface covered by native forest, other studies have shown an opposite trend. Therefore, the capacity of the forest monitoring system to measure the temporal variation in forest areas was evaluated. Specifically, a review of reports and official data of the national forest monitoring system was carried out through the fulfillment of three basic criteria: i) comparability, ii) replicability and iii) quality. According to our results, the Chilean forest monitoring system does not fulfill their basic requirements because: (i) methodologies have not been consistent over time; (ii) it does not provide a baseline of land cover or forest loss that allows comparisons with changing forest area; iii) there is not adequate error estimation and how it can affect the results and analysis of monitoring. The national forest monitoring system requires a redefinition of its aims and methods, guiding them to the long term by convening different stakeholders looking for a national agreement.

Key words: land cover change, land use change, REDD+, deforestation, national forest monitoring.

\section{RESUMEN}

El monitoreo de los bosques es fundamental para la toma de decisiones de manejo y conservación. En Chile, el sistema de monitoreo de bosques se inició en el año 1994, y se ha mantenido mediante la realización de actualizaciones regionales. Esto ha permitido hacer un seguimiento temporal de la superficie, distribución y características del bosque nativo. Sin embargo, mientras las actualizaciones regionales han reportado un continuo aumento del bosque nativo a nivel nacional, otras fuentes han mostrado una tendencia contraria. A raíz de esto, se evaluó la capacidad del sistema de monitoreo forestal para realizar un seguimiento temporal de la superficie de bosques. Específicamente se realizó una revisión de los informes y datos oficiales a través de tres criterios básicos que deben poseer los sistemas de monitoreo forestal: i) comparabilidad, ii) replicabilidad y iii) calidad. De acuerdo con los resultados obtenidos, el sistema de monitoreo de bosques chileno no cumple con estos requisitos mínimos ya que: i) las metodologías no son consistentes en el tiempo, ii) no proporciona una línea base de coberturas del suelo ni de deforestación que permita hacer comparaciones con las tasas de cambio futuras, iii) no existe una estimación adecuada de la incertidumbre de los resultados, ni como ésta puede afectar al monitoreo. El sistema de monitoreo nacional forestal requiere una redefinición de sus objetivos, alcances y métodos, de manera de ofrecer información clara de un recurso clave en la conservación de la biodiversidad y estrategias de mitigación y adaptación al cambio climático.

Palabras clave: cambio de uso del suelo, cambio de cobertura del suelo, REDD+, deforestación, monitoreo nacional forestal, Chile. 


\section{INTRODUCCIÓN}

El monitoreo de los bosques, referido al seguimiento en el tiempo de sus diferentes atributos, es fundamental para la toma de decisiones de manejo y conservación. Representa una tarea de relevancia estratégica para mantener los bienes y servicios que estos proveen, como a su vez la biodiversidad que sustentan (Corona 2016). Corresponde a la fuente de información básica para el desarrollo de políticas públicas, pero a su vez en la evaluación de las mismas a través del territorio a mediano y largo plazo (Holmgren et al. 2007). En un contexto de cambios y compromisos globales, el monitoreo de bosques resulta aún más crítico debido a su relevancia para el desarrollo de estrategias que apuntan a la mitigación y adaptación al cambio climático (Holmgren et al. 2007, MacDicken 2015).

En este ámbito, desde el año 1946, la Organización de las Naciones Unidas para la Alimentación y la Agricultura (FAO) publica el informe Global Forest Resources Assessments (FRA), mediante el cual ha cuantificado y monitoreado la superficie de bosques de la mayoría de países del mundo (FAO 2015). Estos informes, basados en la información suministrada por cada uno de los países miembro de las Naciones Unidas, han sido fundamentales para alertar sobre las pérdidas de bosques a escala global, cifradas en $6 \%$ solo entre 1990 y 2015 (2,4 millones de $\left.\mathrm{km}^{2}\right)$ (Keenan et al. 2015). Bajo este escenario, las Naciones Unidas crearon en el año 2008 el programa Reducing Emissions From Deforestation and Degradation (REDD), en países en vías de desarrollo (Herold y Skutsch 2011). Este programa considera el monitoreo nacional forestal como un eje central en la estrategia de adaptación y mitigación al cambio climático, fomentando su desarrollo en países en los cuales previamente no existía o era muy deficiente (Romijn et al. 2015). Es así como un sistema de monitoreo forestal permite el cumplimiento de diferentes compromisos internacionales, tales como la Convención Marco de las Naciones Unidas sobre el Cambio Climático y el Convenio sobre la Diversidad Biológica, entre otros (FAO 2014a).

FAO (2014a) señala que no existe un método estándar de monitoreo forestal, ya que estos deben adecuarse a la realidad social, técnica y económica de cada país. Sin embargo, señala que existen ciertos requisitos básicos que se deben cumplir, para velar por sus resultados independiente de quién, dónde y cuándo se ejecute. En particular, señala tres requisitos aplicables al monitoreo de la superficie de bosques: i) comparabilidad, referido a la consistencia en el tiempo de la metodología de manera que permita la comparación de datos obtenidos en diferentes momentos; ii) replicabilidad, esto implica que todos los procedimientos técnicos estén justificados en términos científicos y la metodología sea claramente establecida y replicable; iii) calidad de la información obtenida, referido a la exactitud con la cual se realiza la cartografía y la categorización de cada unidad territorial, cuantificada mediante la evaluación del error de las estimaciones.
En el año 1994 se inició en Chile el desarrollo del primer sistema nacional de cartografía de usos y coberturas del suelo, denominado "Catastro y Evaluación de los Recursos Vegetacionales Nativos" (catastro). Este sistema permitió construir una base de datos cartográfica oficial de la superficie de bosques y otros usos y/o coberturas del suelo. Sus resultados permitieron determinar, por primera vez, la superficie y distribución espacial de los bosques nativos a escala nacional, usando una metodología estándar para todo el territorio (CONAF et al. 1999). La realización del catastro posibilitó además la caracterización de los bosques nativos en función de su estructura (e.g. bosque adulto, renoval, bosques achaparrados), tipo forestal, especies dominantes y grado de intervención. El catastro fue diseñado como línea base de una herramienta de seguimiento temporal de la superficie y distribución de los bosques, dando inicio a un sistema cartográfico inédito de monitoreo forestal nacional (CONAF et al. 1999).

El primer informe de cambios temporales en la superficie de bosques se realizó a partir de actualizaciones regionales resumidas en el estudio "Monitoreo de Cambios y Actualizaciones" (CONAF 2011). A base de estas actualizaciones, desde el año 2010 y con un periodo quinquenal se realiza el informe nacional que contribuye al informe global FRA (FAO 2015). En el último reporte para Chile, se indica un aumento neto de la superficie de bosques primarios $^{1}$ desde 4.631 .000 ha en el año 1990 a 5.355 .000 ha en el año 2015, y a su vez, un aumento de otros bosques regenerados de manera natural ${ }^{2}$ de 8.925 .000 a 9.336 .000 ha (FAO 2014b). Estas cifras se contraponen a lo señalado por Miranda et al. (2017), quienes basados en distintos artículos publicados en revistas científicas, entre los años 2006 y 2015, detectaron una tendencia general opuesta para esos años entre las regiones de Valparaíso y Los Lagos. A su vez, las cifras de FAO (2014b), también parecen inconsistentes con las estadísticas nacionales de incendios forestales, ya que según estas, entre los años 1991-2015 se han quemado 270.165 ha de bosque a nivel nacional, considerando solo arbolado nativo (CONAF 2016a).

Si bien en el monitoreo de la cobertura forestal diferentes aproximaciones metodológicas pueden generar resultados dispares, las diversas investigaciones científicas publicadas hasta la fecha, coinciden en determinar una mayor pérdida que ganancia de bosques nativos entre las regiones de Valparaíso y Los Lagos (Heilmayr et al. 2016, Miranda et al. 2017). Las discrepancias en la trayectoria de la superficie de bosques nativos determinadas por trabajos científicos y las estimaciones generadas por FAO (2014b), son completamente opuestas, generando una controversia difícil de resolver en relación a los cambios históricos.

Bosque nativo adulto o achaparrado con una cobertura de copa mayor al $75 \%$ (FAO 2014b)

Bosque nativo adulto con cobertura de copa entre 10 y $50 \%$, bosque nativo renoval de 10 a $100 \%$ de cobertura de copa o bosque adulto renoval con 10 a $100 \%$ de cobertura de copa (FAO 2014b). 
Esta discrepancia, demanda la urgente necesidad de dilucidar la raíz de la divergencia en la trayectoria temporal de las cifras, para así mejorar los mecanismos de monitoreo de bosques.

El objetivo de este estudio es analizar la capacidad del sistema chileno de monitoreo nacional forestal para realizar un seguimiento temporal de la superficie de bosques. En particular, se evalúa el cumplimiento de los tres requisitos mínimos que debe poseer un sistema de monitoreo nacional forestal: comparabilidad, replicabilidad y calidad. De acuerdo a los antecedentes presentados, la hipótesis de partida es que el sistema de monitoreo forestal no permite estimar cambios en la superficie de los bosques debido a que no cumple los requisitos mínimos exigidos para este propósito. Este análisis es recomendado internacionalmente (FAO 2014a) y resulta muy relevante a los 25 años de haberse iniciado el primer sistema de monitoreo de bosques nativos en Chile.

\section{MÉTODOS}

Áreas de estudio. Se analizaron tres fuentes de información distintas que comprenden diferentes áreas de estudio: i) primer informe nacional de "Monitoreo de Cambios y Actualizaciones" (CONAF 2011), en el cual se reúnen las primeras actualizaciones regionales a nivel nacional; ii) informe final "Monitoreo de cambios, corrección cartográfica y actualización del catastro de bosque nativo en las regiones de Valparaíso, Metropolitana y Libertador Bernardo O'Higgins" (CIREN y CONAF 2013); iii) informe final "Monitoreo de cambios, corrección cartográfica y actualización del catastro de los recursos vegetacionales nativos de la región de La Araucanía" (UACh y UFRO 2014). Los informes y cartografía oficial se encuentran disponibles en CONAF (2016b). En la descripción de los métodos para analizar la comparabilidad, replicabilidad y calidad se detallará específicamente el tipo de datos y las zonas de estudio específicas.

Evaluación de la comparabilidad. La comparabilidad del sistema se analizó mediante la descripción de las metodologías utilizadas en el año base del catastro y en las sucesivas actualizaciones regionales, para determinar su consistencia en el tiempo. Un sistema de monitoreo con mediciones comparables no debería sufrir modificaciones en su metodología, de manera que los resultados producidos puedan justificarse en términos científicos (FAO 2014a). En particular se detallaron los datos cartográficos utilizados para realizar los mapas, escalas, unidad mínima cartografiable (UMC) y las definiciones de los principales usos/coberturas del suelo.

Evaluación de la replicabilidad. Este requisito se evaluó en función de la disponibilidad de información, protocolos y métodos claros y explícitos de las actualizaciones regionales y las modificaciones posteriores a los datos original- mente obtenidos. Adicionalmente, para evaluar los efectos que han tenido las modificaciones realizadas a los datos originalmente generados, se compararon las cifras de la cartografía original con las cifras obtenidas una vez realizadas las modificaciones posteriores. A su vez, se analizó la naturaleza de estas modificaciones, es decir, si corresponde a una homogenización cartográfica, o también a un cambio en la asignación de coberturas del suelo. Para estos análisis, se utilizó como caso de estudio la cartografía generada en la primera y segunda actualización de la región de La Araucanía, CONAF et al. (2009) y UACh et al. (2014), respectivamente. Se comparó la categoría de cobertura del suelo definida para cada polígono en la actualización original con la definida para el mismo polígono después de las modificaciones realizadas a la actualización original u "homogenización cartográfica". Este proceso se realizó en 450 polígonos clasificados como de tipo de cambio "14", que corresponde a "corrección en la fotointerpretación o descripción original de acuerdo con UACh et al. (2014). Los polígonos fueron seleccionados de manera aleatoria estratificada. Se estratificó en función de tres rangos de superficie: 0,5 a 20 ha, 20 a 100 ha, y mayor de 100 ha, seleccionando la misma cantidad de polígonos en cada estrato. Adicionalmente se analizaron los posibles efectos de estas modificaciones sobre la superficie originalmente estimada de los diferentes tipos forestales, lo que se evaluó utilizando los polígonos que permanecieron como bosque nativo después de las modificaciones realizadas a la actualización original.

Evaluación de la calidad. La calidad del sistema de monitoreo se refiere a la exactitud con la cual se categorizó cada clase de uso y/o cobertura del suelo individualmente, tanto en la cartografía base como en las actualizaciones, así como al manejo y documentación de errores y fuentes de error de acuerdo a las recomendaciones de FAO (2014a). Las fuentes de error pueden provenir de la delimitación de cada clase de uso y/o cobertura del suelo, la posición geográfica, y la definición y asignación de las coberturas del suelo, entre otros (Giri 2012). Sin embargo, en este estudio se analizó la exactitud con la cual se asignan los diferentes usos/coberturas del suelo (e.g. bosque nativo, plantaciones forestales, praderas) utilizando las estimaciones del error reportado por los informes regionales. Se consideró dentro de la evaluación, la existencia o no, de algún parámetro de medición de error, y de existir, se reportó el valor de dicha medición. En la evaluación de la categorización de usos y/o coberturas del suelo, el indicador más ampliamente utilizado es la exactitud global, que representa el porcentaje de aciertos en relación a la categoría asignada y la observada en la evaluación en terreno u otra fuente (Congalton 1991). También se recomienda evaluar la exactitud por cada categoría de usos y/o coberturas y en función de los diferentes niveles de agrupación. En particular, se evaluó la calidad de cada actualización de acuerdo a la evaluación de la exactitud a diferentes niveles de agrupación de las 
coberturas del suelo, registrando la exactitud global y la exactitud por cobertura.

Para clasificar el tipo de uso/cobertura del suelo, tanto en el catastro original como en las actualizaciones, los equipos ejecutores de las actualizaciones regionales definieron las categorías "uso", "subuso" y "estructura". Dentro de la categoría de uso "bosques", se definieron los subusos: i) plantaciones, ii) bosque nativo y iii) bosques mixtos. A su vez, dentro de los bosques nativos se definieron como estructuras las categorías: i) bosque nativo adulto, ii) renoval, iii) bosque nativo adulto-renoval y iv) bosques achaparrados.

\section{RESULTADOS}

Evaluación de la comparabilidad. CONAF (2011) reporta los resultados de la comparación del catastro original (1994-1998) con las primeras actualizaciones regionales. Dichas actualizaciones fueron realizadas entre los años 2000 y 2010, reportando cambios en periodos de entre 5 a 14 años para las distintas regiones (cuadro 1). CONAF (2011) reporta a su vez cambios en la metodología utilizada para la elaboración del catastro original y las actualizaciones regionales. En particular, en las primeras actualizaciones regionales se observa un cambio en la UMC, es decir en la superficie mínima de bosque identificada. En las sucesivas actualizaciones regionales, la UMC es nuevamente modificada en las regiones de Valparaíso, Metropolitana y Libertador Bernardo O'Higgins a 0,5 ha en las áreas cubiertas por bosque, no indicando su valor para otras regiones (cuadro 1).

Para subsanar las diferentes UMC, los equipos ejecutores realizaron homogenizaciones cartográficas (e.g. UACh et al. 2014). Lo anterior, significa que se modificó la forma de los polígonos originales, y se modificó también la categoría de uso y/o cobertura del suelo de ciertos polígonos en función de la nueva fotointerpretación. Dichos cambios no solo se han realizado en el catastro original, sino también en las actualizaciones regionales para su comparación en fechas sucesivas. En la región de La Araucanía, debido a la homogenización cartográfica del catastro original (1993), se produjo un aumento de 68.637 ha de bosque nativo, lo que representa el doble de lo determinado como cambio entre los años 1993 (homogeneizado cartográficamente) y la primera actualización regional el año 2007 (cuadro 2A). A su vez, la actualización regional del año 2007 también fue homogeneizada cartográficamente, lo que significó un aumento de 43.400 ha de bosque nativo respecto a lo reportado inicialmente por CONAF et al. (2009), 20 veces más que la superficie de cambio reportada entre los años 2007 (homogeneizado cartográficamente) y la última actualización (UACh et al. 2014) (cuadro 2A). Una tendencia contraria se observa con las plantaciones forestales de especies exóticas, las cuales presentan una menor superficie en cada homogenización cartográfica en comparación con la reportada inicialmente (cuadro 2A). En el cuadro 2B se observan las diferencias que ha implicado la homogenización cartográfica realizada en el catastro y sus actualizaciones en la denominada macro zona central, correspondiente a las regiones de Valparaíso, Metropolitana y Libertador Bernardo O'Higgins. Todas las modificaciones realizadas han implicado un aumento de la superficie de bosque nativo reportada en cada homogenización cartográfica con respecto a la estimada originalmente.

En cuanto a los cambios temporales de superficie en el bosque nativo, el mayor cambio es el reportado entre la actualización homogeneizada del año 2001 y la actualización realizada para el año 2011, en las regiones de Valparaíso, Metropolitana y Libertador Bernardo O'Higgins. Se señala un aumento neto del bosque nativo de, aproximadamente, 900.000 ha. Esta superficie representa un $340 \%$ del total de bosque nativo reportado para el año 2001 después de ser homogeneizada (cuadro 2B).

Evaluación de la replicabilidad. En todas las actualizaciones regionales se ha realizado la denominada "homogenización cartográfica" que corresponde a una "corrección en la fotointerpretación o descripción original”. Estas modificaciones se han realizado, tanto para el catastro original como para las actualizaciones regionales. Los métodos con los cuales se realizan estas correcciones o homogenizaciones cartográficas no se detallan en los informes. Sin embargo, de los informes se puede deducir que estas modificaciones incluyen: i) generación de nuevos polígonos; ii) re-digitalización de polígonos, iii) cambio de categoría de uso y/o cobertura del suelo, y iv) cambios en el tipo forestal. Por ejemplo, en la región de La Araucanía, de acuerdo con UACh et al. (2014), del total de cambios realizados en el proceso de "homogenización cartográfica", 478.472 ha representan cambios por la "corrección en la fotointerpretación o descripción original", lo que corresponde a un $58,1 \%$ de los cambios temporales entre los años 2007 y 2013 , y que representan el $15 \%$ de la superficie de la región. También se constató la re-categorización de polígonos desde otras coberturas del suelo a bosque nativo, lo que significó un aumento del bosque nativo original (cuadro 2A). Los nuevos fragmentos de bosque nativo agregados a la actualización original del año 2007 no presentan una característica común de tamaño, forma o categoría inicial (figura 1).

En la región de La Araucanía, las mayores modificaciones al realizar las homogenizaciones cartográficas se produjeron por una re-digitalización de los polígonos o cambios en la densidad del bosque, permaneciendo en la misma categoría de cobertura del suelo. En la figura 1, se observa un ejemplo de las áreas con bosque nativo inicialmente definidas en el año 2007 en comparación con aquellas agregadas en base a las homogenizaciones cartográficas para el mismo año. Estas modificaciones de categoría se realizaron en un $45 \%$ de los polígonos analizados (cuadro 3A). Las mayores modificaciones se concentraron en los bosques adultos y renovales. En particular, $47 \%$ de 


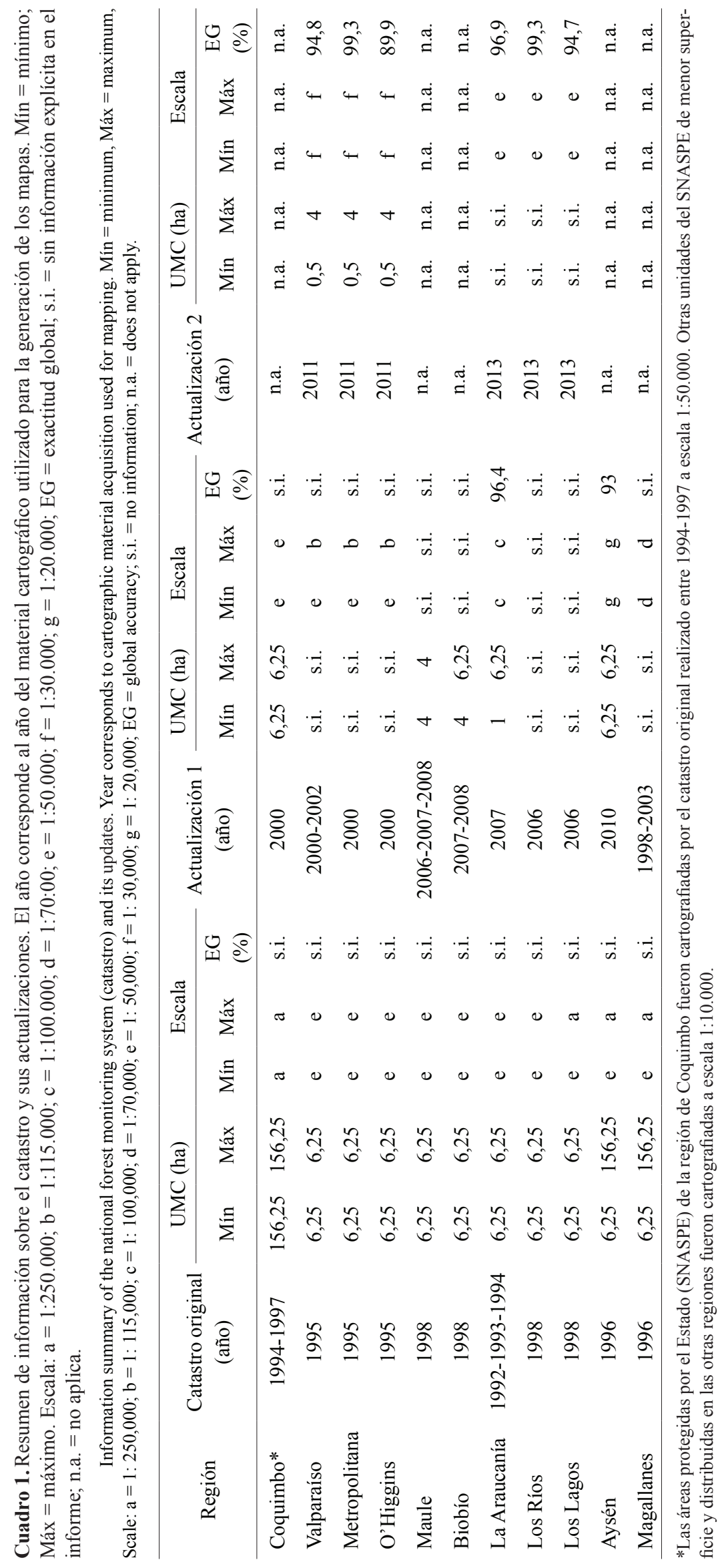


Cuadro 2. Diferencia en la superficie (ha) de plantaciones forestales y bosques nativos entre los datos del catastro original, los datos modificados del catastro original, la primera actualización y las modificaciones realizadas a los datos originales de la primera actualización y la segunda actualización de las regiones: A) La Araucanía y B) Valparaíso, Metropolitana y Libertador Bernardo O’Higgins. $\mathrm{HC}=$ Homogenización cartográfica de los datos originales. ${ }^{*}$ El total de bosque nativo corresponde a la suma de bosque adulto, reno-

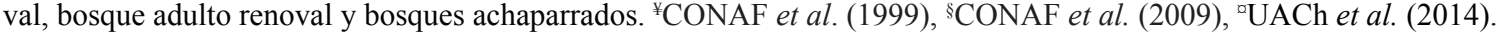

Differences in forest area (ha) covered by tree plantations and native forest among the original dataset of the national forest monitoring system (catastro), the modified data of the original "catastro", first updates and the modifications of the original data of the first update: A) La Araucanía and B) Valparaíso, Metropolitana and O'Higgins Regions. $\mathrm{HC}=$ Cartographic homogenization of original data. *Total of native forest corresponds to the sum of old growth forest, second growth forest, old and second growth forest and Krumholz. ${ }^{*} \mathrm{CONAF}$ et al. (1999), ${ }^{8} \mathrm{CONAF}$ et al. (2009), ${ }^{\mathrm{a} U A C h}$ and UFRO (2014). ${ }^{\bullet}$ Primeras actualizaciones regionales Valparaíso, Metropolitana y LB O’Higgins (CONAF 2011). ${ }^{\square}$ CIREN y CONAF (2013).

\begin{tabular}{|c|c|c|c|c|c|}
\hline \multicolumn{6}{|l|}{ A) La Araucanía } \\
\hline \multirow[b]{2}{*}{ Categorías } & \multicolumn{2}{|c|}{1993} & \multicolumn{2}{|c|}{2007} & \multirow{2}{*}{$\begin{array}{l}2013 \\
\text { Original }\end{array}$} \\
\hline & Original $^{*}$ & $\mathrm{HC}^{\S}$ & Original $^{\S}$ & $\mathrm{HC}^{\mathrm{a}}$ & \\
\hline Plantaciones & 359.906 & 351.331 & 572.185 & 566.438 & 632.289 \\
\hline Bosques mixtos & 19.074 & 30.539 & 28.952 & 47.683 & 47.639 \\
\hline Bosque adulto & 286.304 & 286.318 & 277.914 & 274.157 & 275.196 \\
\hline Renoval & 449.447 & 500.697 & 475.409 & 482.903 & 483.275 \\
\hline Bosque adulto Renoval & 90.565 & 101.812 & 96.643 & 119.047 & 120.173 \\
\hline Bosques Achaparrados & 82.185 & 88.311 & 87.238 & 85.766 & 85.508 \\
\hline Total bosque nativo* & 908.501 & 977.138 & 937.204 & 961.873 & 964.153 \\
\hline \multicolumn{6}{|c|}{ B) Valparaíso, Metropolitana y Libertador Bernardo O'Higgins } \\
\hline \multirow[b]{2}{*}{ Categorías } & \multicolumn{2}{|c|}{1995} & \multicolumn{2}{|c|}{2000} & 2011 \\
\hline & Original $^{*}$ & $\mathrm{HC}^{\bullet}$ & Original $^{\bullet}$ & $\mathrm{HC}^{\square}$ & Original $^{\square}$ \\
\hline Plantaciones & 170.641 & 166.647 & 182.756 & 189.274 & 208.475 \\
\hline Bosques mixtos & 1.687 & 1.830 & 1.186 & 1.462 & 1.489 \\
\hline Bosque adulto & 3.334 & 3.916 & 3.649 & 4.825 & 8.118 \\
\hline Renoval & 298.311 & 315.846 & 311.909 & 367.689 & 1.263 .100 \\
\hline Bosque adulto Renoval & 4.317 & 10.680 & 9.505 & 22.245 & 35.069 \\
\hline Bosques Achaparrados & 819 & 11.751 & 11.889 & 11.930 & 1.092 \\
\hline Total bosque nativo* & 306.780 & 342.192 & 336.951 & 406.689 & 1.307 .379 \\
\hline
\end{tabular}

los polígonos modificados y originalmente categorizados como bosque nativo adulto fueron cambiados de categoría. Un $30 \%$, aproximadamente, cambió a renovales y el restante a otros usos y/o coberturas de suelo. En cuanto a los renovales, el $60 \%$ mantuvo dicha clasificación. La mayor parte de los renovales que no se clasificaron como tales en el proceso de homogeneización cartográfica fueron recategorizados a otros usos y/o coberturas del suelo. En las plantaciones de especies forestales exóticas, el $63 \%$ mantuvo esta categoría, siendo modificadas una mayor parte a otros usos y/o coberturas del suelo y menos del $15 \%$ a alguna de las categorías de bosque nativo. Por otro lado, solo el $33 \%$ de la superficie de matorrales conservó su categoría después de dichas modificaciones, cambiándolo principalmente a plantaciones de especies forestales exóticas (cuadro 3).
De los 450 polígonos seleccionados, un $62 \%$ fue inicialmente categorizado como bosque nativo adulto o renoval en el año 2007 (cuadro 3). De estos, $91 \%$ se mantuvo categorizado como bosque nativo después de las modificaciones. Cada uno de estos polígonos de bosque nativo fue categorizado inicialmente en diferentes tipos forestales en una proporción del $49 \%$ Roble-Raulí-Coigüe, $21 \%$ Araucaria, $14 \%$ Coigüe-Raulí-Tepa, $11 \%$ Lenga y $5 \%$ Siempreverde. En términos netos, Roble-Raulí-Coigüe, Araucaria, Lenga y Siempreverde obtuvieron un $3 \%, 4 \%$, $23 \%$ y $29 \%$ de nuevos polígonos, respectivamente, que en parte habrían sido recategorizados desde Coigüe-RaulíTepa. Este tipo forestal tuvo una disminución del $42 \%$ en el número de polígonos.

Un $68 \%$ de los polígonos mantuvo el tipo forestal inicialmente definido. Sin embargo, todos los tipos forestales 


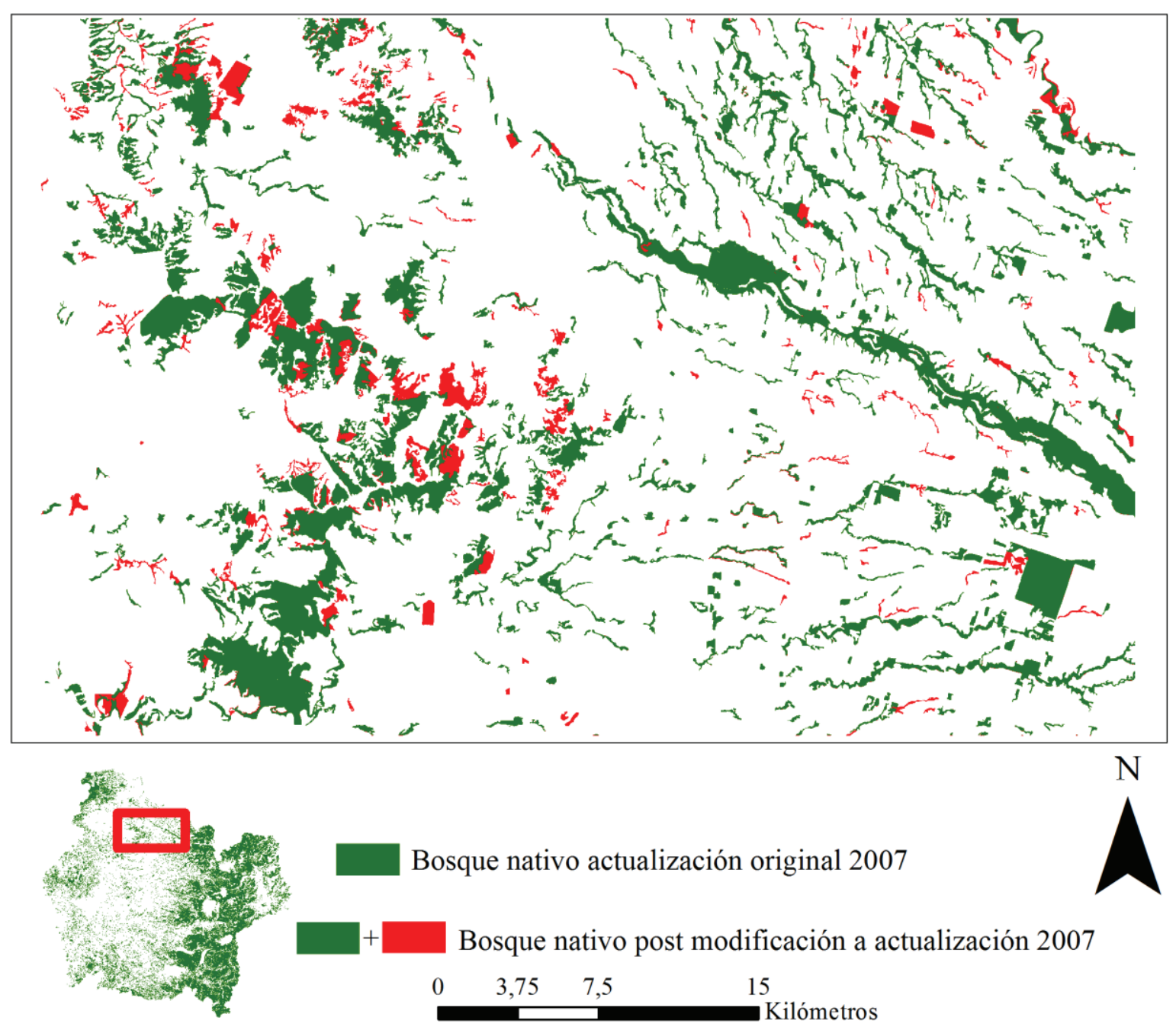

Figura 1. Ejemplo de modificaciones del bosque nativo en la región de La Araucanía a partir de la homogenización cartográfica realizada a la actualización de 2007 (Fuente: CONAF et al. (2009), UACh et al. (2014)).

Example of native forest changes in La Araucanía Region from the cartographic homogenization made to the updating of the national forest monitoring system developed in 2007 (Source: CONAF et al. (2009), UACh y UFRO (2014)).

sufrieron modificaciones en diferentes proporciones. La mayor permanencia fue del tipo forestal Roble-Raulí-Coigüe con un $78 \%$, seguido por Araucaria con un $75 \%$, y la menor del tipo forestal Coigüe-Raulí-Tepa con un $26 \%$ de permanencia (cuadro 3). Por otro lado, una alta proporción de polígonos descritos con algún tipo forestal en la homogenización cartográfica, fueron reclasificados a partir de otras clases de uso y/o cobertura del suelo. Por ejemplo, $49 \%$ de los polígonos clasificados inicialmente como matorrales fueron posteriormente clasificados como algún tipo forestal. Algo similar ocurre con las plantaciones forestales, aunque en menor proporción, donde un $12 \%$ fue reclasificado en algún tipo forestal. Para la categoría "Protección", inicialmente definida sin tipo forestal, al $69 \%$ se le atribuyó alguno de los tipos forestales posteriormente a las modificaciones.
Evaluación de la calidad. No fue posible evaluar la exactitud del catastro original a ningún nivel ya que no se tuvo acceso a la evaluación de la exactitud global (cuadro 1). En las primeras actualizaciones regionales sólo se reporta la exactitud para la región de Aysén. En las actualizaciones actuales en las regiones de Valparaíso, Metropolitana, Libertador Bernardo O'Higgins, La Araucanía, Los Ríos y Los Lagos se ha determinado la exactitud en tres niveles de agrupación diferente (cuadro 4). Sin embargo, no se evalúa la exactitud de la asignación de tipos o subtipos forestales. Tampoco se reevaluó la exactitud de las actualizaciones regionales después de realizar la homogenización cartográfica.

La exactitud en las actualizaciones regionales actuales a nivel de Uso varía entre un 89,9 y el $99,3 \%$, la cual es en general mayor que las de Subuso y esta, a su vez, ma- 
Cuadro 3. Modificación por medio de "homogenización cartográfica" de A) uso y/o cobertura del suelo y B) tipo forestal, a raíz de las homogenizaciones cartográficas realizadas a la primera actualización de la región de La Araucanía. Los cuadros representan una matriz de cambio desde la actualización original (columna 1) a la original homogeneizada (fila 1). Los valores representan el número de polígonos a los cuales se les recategorizó de uso y/o cobertura del suelo (A) o de tipo forestal (B). BNad: Bosque nativo adulto. Mtr: Matorral. Pl: Plantaciones forestales. Rn: Renoval. Ar: Araucaria. CoRaTe: Coigüe-Raulí-Tepa. Le: Lenga. Ro-Ra-Co: Roble-RaulíCoigüe. Sv: Siempreverde.

Modification of A) land use/land cover and B) forest type, as result of the "cartographic homogenization" made to the first update of La Araucanía Region. Tables represent a matrix change from the original update (column 1) to the original homogenized (row 1). Values represent the number of polygons to which they were re-categorized to different use and/or land cover (A) or forest type (B). BNad: Old growth forest. Mtr: Shrubs. Pl: Forest tree plantations. Rn: Second growth forest. Ar: Araucaria. CoRaTe: Coigüe-Raulí-Tepa. Le: Lenga. Ro-Ra-Co: Roble-Raulí-Coigüe. Sv: Evergreen.

\begin{tabular}{|c|c|c|c|c|c|c|c|}
\hline \multirow[t]{2}{*}{$\mathrm{A}$} & \multirow{2}{*}{$\begin{array}{l}\text { Uso/Cobertura } \\
\text { Original } 2007\end{array}$} & \multicolumn{5}{|c|}{ Homogenización cartográfica } & \multirow[t]{2}{*}{ Permanencia $(\%)$} \\
\hline & & $\mathrm{BNad}$ & Mtr & Otros & $\mathrm{Pl}$ & $\mathrm{Rn}$ & \\
\hline & BNAd & 60 & 8 & 13 & 0 & 32 & 53 \\
\hline & Mtr & 3 & 8 & 6 & 6 & 1 & 33 \\
\hline & Otros & 1 & 15 & 38 & 13 & 15 & 46 \\
\hline & Pl & 2 & 1 & 15 & 42 & 7 & 63 \\
\hline & $\mathrm{Rn}$ & 17 & 9 & 32 & 8 & 101 & 60 \\
\hline \multirow[t]{7}{*}{$\mathrm{B}$} & Tipo Forestal & \multicolumn{5}{|c|}{ Homogenización cartográfica } & Permanencia (\%) \\
\hline & Original 2007 & $\mathrm{Ar}$ & CoRaTe & $\mathrm{Le}$ & RoRaCo & Sv & \\
\hline & $\mathrm{Ar}$ & 36 & 1 & 9 & 2 & 0 & 75 \\
\hline & CoRaTe & 3 & 8 & 2 & 15 & 3 & 26 \\
\hline & Le & 4 & 0 & 14 & 6 & 0 & 58 \\
\hline & RoRaCo & 3 & 9 & 6 & 86 & 6 & 78 \\
\hline & Sv & 0 & 0 & 0 & 4 & 8 & 67 \\
\hline
\end{tabular}

Cuadro 4. Evaluación de la exactitud (\%) a nivel de Uso, Subuso y Estructura en las regiones en las cuales se ha realizado este análisis. Fuente: CONAF (2016c).

Accuracy assessment at Use, Subcategory of Use and Structure levels in the regions where this analysis was carried out (Source: CONAF (2016c)).

\begin{tabular}{lcccc}
\hline Región & Año & Uso & Subuso & Estructura \\
\hline Valparaíso & 2013 & 94,8 & 87,6 & 87,3 \\
Metropolitana & 2013 & 99,3 & 94,5 & 91,3 \\
O’Higgins & 2013 & 89,9 & 86,6 & 86,4 \\
La Araucanía & 201 & 96,9 & 97,5 & 91,9 \\
Los Ríos & 2014 & 99,3 & 99,6 & 98,4 \\
Los Lagos & 2014 & 94,7 & 91,8 & 87,4 \\
Aysén & 2011 & 93,0 & 89,0 & 83,0 \\
\hline
\end{tabular}

yor que la de Estructura. Sin embargo, en las regiones de La Araucanía y Los Ríos, la exactitud de los Subusos es mayor que la de Usos, alcanzando un 97,5 y un 99,6 \%, respectivamente.

\section{DISCUSIÓN}

El sistema de monitoreo de bosques en Chile no cumple con algunos requisitos fundamentales que debe tener un sistema de monitoreo forestal nacional. Ninguno de los aspectos evaluados se cumplen satisfactoriamente por las razones siguientes: i) la metodología no es consistente a través del tiempo, lo que hace que sea un sistema no comparable; ii) no provee una línea base de coberturas del suelo ni tampoco de deforestación que permita hacer comparaciones con las tasas de cambio futuras, lo que deriva en un sistema no replicable debido a continuos cambios en los datos y en la falta de información sobre cómo se realizan estos cambios; iii) la estimación de la incertidumbre de los resultados se cumple de manera parcial, en particular en los últimos años, sin embargo, no se detalla cuáles pueden ser las principales fuentes de error, ni cómo la incertidumbre se integra y puede afectar al monitoreo. Por otro lado, no se mide la incertidumbre para todos los atributos que se consideran en el sistema, como por ejemplo en los tipos y subtipos forestales, por lo tanto, la estimación de los cambios en la superficie de bosque nativo en Chile entre 1999 y 2015 encuentra serias limitaciones. Las actualizaciones y monitoreo realizadas y ordenadas por ley para este propósito, impiden determinar cuál ha sido efectivamente el cambio en la superficie de los bosques nativos. 
La presente investigación da cuenta de que los diversos cambios metodológicos en las actualizaciones del catastro han influido en la estimación del cambio temporal y espacial de la superficie de bosques nativos. Por ejemplo, CONAF (2011) atribuye a los cambios metodológicos la principal razón de la percepción de los cambios de superficie de usos y/o coberturas del suelo. En particular, se señala: "la variación de superficie que experimentan los diferentes subusos de bosque entre lo señalado en el catastro original con base año 1997 y las actualizaciones regionales, no necesariamente corresponden a un aumento o disminución real de dicha superficie. Esta variación en los usos se explica principalmente por una mejora sustancial en la obtención y procesamiento de los materiales cartográficos digitales (fotografías aéreas, imágenes satelitales), lo que ha permitido, a través de los monitoreos realizados, disminuir la Unidad Mínima Cartografiable de 6,25 y 125 ha en algunas zonas, a 4 y 6,25 ha, respectivamente". Por otro lado, más recientemente, los cambios en los criterios para la definición de bosque derivaron en un notable aumento en la estimación de su superficie para las regiones de Valparaíso, Metropolitana y Libertador Bernardo O'Higgins" (CIREN y CONAF 2013). En las últimas actualizaciones, se consideró bosque nativo a cualquier área con una cobertura arbórea de un $10 \%$, mientras que el año 2001 esta cobertura debía tener un mínimo del $25 \%$. Ello generó un aumento en la estimación de la superficie de bosque nativo desde las clases de uso definidas como praderas y matorrales, triplicando su superficie (CIREN y CONAF 2013).

En otro ámbito, tanto el catastro original como las actualizaciones han sido modificados en sus cifras originales para su comparación con las actualizaciones más recientes. En consecuencia, no se cuenta con una línea base de coberturas del suelo ni de deforestación que permita hacer comparaciones con las tasas de cambio futuras. Estas modificaciones son mayores que los mismos cambios temporales reportados (cuadro 2). La estimación de la incertidumbre en el cálculo de las superficies de bosques es otro aspecto débil del actual sistema de monitoreo. En las primeras actualizaciones regionales esta estimación fue reportada únicamente para dos de las 11 regiones analizadas (cuadro 1). Además, al realizar modificaciones a las actualizaciones no se re-evalúa la exactitud de los resultados. Por ejemplo, para la primera actualización de la región de La Araucanía se reportó un 96,4 \% de exactitud en la categorización de usos de suelo, pero no se señala si con las modificaciones este valor varía. Las actualizaciones posteriores a 2011 si cuentan con la evaluación de la incertidumbre, pero en el caso de realizar una "homogenización cartográfica" se debe re-evaluar la estimación del error. No fue posible establecer si las fuentes de datos para realizar las validaciones y cálculo de exactitudes son realmente independientes de los datos usados para construir los mapas de cada año. Este aspecto es altamente recomendable para evitar enmascarar errores en la atributación de los polígonos que se mantienen en la definición de los polígonos de validación, elevando las exactitudes obtenidas en las matrices de confusión.

Estas inconsistencias metodológicas del sistema de monitoreo nacional representan un problema local, pero con consecuencias globales. Por ejemplo, y a pesar de lo expuesto anteriormente, Chile en su informe para FAO (FAO 2015) reporta un aumento de los bosques naturales desde 1990 hasta 2015 , de 13.556 .000 ha a 14.691 .000 ha. Con estos datos, se posiciona dentro de un pequeño grupo de países donde ha aumentado la superficie de bosques naturales en las últimas décadas. Por otro lado, Chile se ha posicionado en el grupo de países con una adecuada calidad de monitoreo. Ello quiere decir que la fuente de datos con la cual se elaboró el informe es reciente (=10 años), y se realizó a través de un inventario forestal nacional o teledetección con verificación sobre el terreno, o bien, datos proporcionados por instituciones oficiales o programas de inventarios forestales nacionales sucesivos y compatibles (FAO 2014b). Sin embargo, y en función de lo expuesto, la capacidad del sistema de monitoreo de proveer información confiable sobre cambios en la superficie de bosques necesita una revisión.

A pesar de las sucesivas modificaciones realizadas a las actualizaciones regionales, estas no se expresan en los informes FAO (2010) y FAO (2014b). Por ejemplo, en FAO (2010), se reportan las mismas cifras de bosque primario y otros bosques regenerados naturalmente para los años 1990, 2000, 2005 y 2010 que en FAO (2014b). Cada actualización regional ha sufrido modificaciones en las cifras, por lo que sería esperable que estos valores fueran corregidos no solo para el análisis de cambio regional, sino también en las cifras globales reportadas cada 5 años. Otro aspecto a considerar es que para el informe FAO (2014b) solo se utilizaron las actualizaciones de Aysén del año 2010 y de la macrozona central de 2011 (cuadro 1). Si bien, para Aysén se reportó un leve aumento del bosque nativo, para la macrozona central de Chile, este aumento fue estimado en 900.000 ha, que provienen principalmente de renovales dominados por Acacia caven (Mol.) Mol. recategorizados como bosque. Sin embargo, en FAO (2014b) se reporta un aumento del bosque primario desde 4.439 .000 ha el año 2010 a 5.355 .000 ha el año 2015. Es decir, el aumento de bosque nativo provocado por el cambio en la definición de bosque en CIREN y CONAF (2013) con respecto a las versiones anteriores, definidos en el mismo como renovales, se reporta en FAO (2014b) como un aumento en los bosques primarios.

Las inconsistencias metodológicas estudiadas y la consecuente necesidad de mejorar la metodología, definiciones y tecnologías, no solo es un desafío para Chile. Por ejemplo, a partir principalmente de los informes nacionales, FAO (2015) reporta 25 años de cambios en los bosques a escala global. Esta información no se sustenta en métodos homogéneamente definidos, derivando por tanto en resultados variables y escasamente fiables. Debido a cambios en las definiciones y evaluaciones en los monitoreos nacionales, la información publicada por los diversos informes de la FAO, en especial en el último período, no puede ser com- 
parada, lo cual es reconocido por sus mismos autores (MacDicken 2015). Un aspecto clave para cualquier sistema de monitoreo de cambios de uso y/o cobertura del suelo es determinar una línea base de pérdida de bosque, con la cual comparar futuras tasas de cambio, y además, proveer estimaciones de la incertidumbre (Pelletier y Goetz 2015). Sin embargo, también se han revelado importantes diferencias existentes entre países en cuanto a la calidad de la información disponible, dificultando los esfuerzos globales para monitorear el estado de los bosques (Romijn et al. 2015).

Lineas generales para el monitoreo de bosques nativos en Chile. Las debilidades encontradas en el actual sistema de monitoreo hace necesaria una discusión crítica sobre las actuales metodologías y protocolos de cuantificación de la superficie boscosa de manera de alcanzar adecuados estándares internacionales. No obstante que CONAF ha asumido la compleja tarea de coordinar y sacar adelante este sistema, es responsabilidad de todos los actores relacionados con el manejo y conservación de los bosques, lograr tener un sistema de monitoreo acorde a las necesidades nacionales. Aún más, en un contexto de cambios globales, donde obtener información fiable será cada vez más necesario y exigible. Por lo pronto, la mejora del sistema de monitoreo se encuentra ausente de las prioridades del sector forestal, dejándolo fuera de las políticas forestales establecidas hasta 2035 (CONAF y Ministerio de Agricultura 2016).

A partir de los principios del monitoreo forestal nacional (FAO 2014a) se desprenden algunos lineamientos generales para el monitoreo de bosques nativos en Chile. Considerando los principios de gobernanza y alcance, sería necesaria la constitución de un panel de especialistas de diferentes instituciones gubernamentales y académicas. Este panel de carácter permanente debiera definir los objetivos y alcances del sistema de monitoreo, además de la definición de la metodología de monitoreo de usos y/o coberturas del suelo, registro de los cambios en la cobertura forestal, así como, la validación de las cifras obtenidas mediante la actualización y monitoreo de la cobertura de los bosques nativos y otros usos del suelo. A su vez y siguiendo el principio del diseño se debiese analizar y establecer criterios necesarios para asegurar la comparabilidad, replicabilidad y calidad de los datos. Es posible que cada región o zona geográfica tenga singularidades dependiendo de las coberturas y usos del suelo dominantes, topografía y clima que definirá entre otras cosas las UMC, frecuencia de monitoreo, estados de desarrollo y estructuras a reconocer, por lo que el panel debería estar compuesto al menos por un integrante por región. De esta forma, se estarían aprovechando e integrando las capacidades en el monitoreo de la cobertura boscosa que se encuentran dispersas en diferentes instituciones, sin descuidar la integración de temas emergentes locales o nacionales, permitiendo a su vez una revisión periódica del sistema.

Es urgente mejorar el sistema de monitoreo de los bosques dada la alta tasa de degradación del bosque nativo (Miranda et al. 2017) y los complejos escenarios de cam- bio global. En un periodo de 5-10 años, se debiera lograr una mejora substancial a nivel nacional. Las mejoras deben buscar la obtención de mayor información, con mayor nivel de detalle y calidad. Estos cambios pueden provenir a su vez de nuevos avances tecnológicos o de nuevas perspectivas en cuanto al manejo forestal, reconsiderando los objetivos y alcances esperados (Corona 2016). Esto necesariamente debe llevar a la reevaluación de los métodos utilizados e incluso de sus objetivos, ampliándolos o redireccionándolos en función de nuevas necesidades. Sin embargo, esto no puede generar información incompatible entre diferentes periodos de análisis. Es por ello, y siguiendo el principio acerca de los datos, que las metodologías deben estar claramente explicitadas al menos para el año inicial y el siguiente, en lo referido a la definición de coberturas y/o usos del suelo, fuentes de información (e.g. imágenes satelitales), categorización/clasificación, sistema de muestreo, método de validación, estimación de la incertidumbre y otros aspectos relevantes. La incorporación de nuevas tecnologías o métodos de monitoreo debieran contar con un periodo piloto de evaluación. Para ello, la ley de bosque nativo puede establecer nuevas líneas de investigación que permitan nutrir al panel de expertos de experiencias locales con la adaptación de estas nuevas técnicas. Las definiciones específicas del sistema de monitoreo nacional deberán ser limitadas por los organismos pertinentes, en función a lo que como sociedad se proyecte de sus recursos forestales.

Si bien, este estudio se acotó a las variables asociadas a la superficie de bosques, también deben incorporarse otros mecanismos de monitoreo forestal multipropósito, tales como biodiversidad, funcionalidad ecosistémica, grado de degradación, sanidad, flujos de carbono, entre otros (FAO 2014a, Corona 2016).

\section{CONCLUSIONES}

La estimación de cambios en la cobertura de bosque nativo encuentra limitaciones, generando incertidumbre y controversia entre los diferentes actores. Los resultados muestran que los cambios en la superficie de los bosques nativos se deben principalmente a cambios metodológicos y no a cambios reales. Es necesario redefinir los objetivos del sistema de monitoreo nacional, su alcance esperado y la metodología para alcanzarlos. Todo ello se debe realizar dentro de un contexto de un análisis crítico, discusión y acuerdo fundado en la ciencia y técnica, y experiencia internacional que permita definir las líneas generales y realizar una evaluación continua de los resultados. Estas medidas están orientadas a constituir un sistema robusto de monitoreo nacional que permita enfrentar los desafíos que imponen los rápidos cambios globales.

\section{AGRADECIMIENTOS}

Los autores agradecen el apoyo financiero de esta investigación a los proyectos: CONICYT/FONDAP N ${ }^{\circ} 15110009$, 
FONDECYT No 1141294, FONDECYT N 1171445. También se reconoce el apoyo del Programa de Becas de Doctorado Nacional de CONICYT N 21140409 y el proyecto FONDEF IdeaCA13I10276. AP agradece el financiamiento de los proyectos ICM P05-002 y CONICYT PFB-23. Los autores agradecen a los cuatro revisores anónimos quienes colaboraron sustancialmente a mejorar el manuscrito.

\section{REFERENCIAS}

CIREN (Centro de Información de Recursos Naturales, CL), CONAF (Corporación Nacional Forestal, CL). 2013. Informe técnico final proyecto: Monitoreo de cambios, corrección cartográfica y actualización del catastro de bosque nativo en las regiones de Valparaíso, Metropolitana y Libertador Bernardo O'Higgins. Santiago, Chile. 130 p.

CONAF (Corporación Nacional Forestal, CL). 2011. Catastro de los recursos vegetacionales nativos de Chile: Monitoreo de cambios y actualizaciones período 1997 - 2011. Santiago, Chile. 28 p.

CONAF (Corporación Nacional Forestal, CL), CONAMA (Comisión Nacional del Medio Ambiente, CL), BIRF (Banco Internacional de Reconstrucción y Fomento, US), UACH (Universidad Austral de Chile), PUC (Pontificia Universidad Católica de Chile), UC (Universidad Católica de Chile). 1999. Catastro y evaluación de recursos vegetacionales nativos de Chile: Informe nacional con variables ambientales. Santiago, Chile. 89 p.

CONAF (Corporación Nacional Forestal, CL), CONAMA (Comisión Nacional del Medio Ambiente, CL), Universidad Austral de Chile. 2009. Catastro de uso del suelo y vegetación. Monitoreo y actualización región de La Araucanía. Periodo 1993-2007. 29 p.

CONAF (Corporación Nacional Forestal, CL). 2016a. Ocurrencia y daño de incendios forestales. Acumulado temporadas 1991 - 2015. Gerencia Protección Contra Incendios Forestales. Estadísticas-Julio 2015. Consultado 3 sep. 2016. Disponible en http://www.conaf.cl/incendios-forestales/ incendios-forestales-en-chile/estadisticas-historicas/

CONAF (Corporación Nacional Forestal, CL). 2016b. Sistema de Información Territorial. Consultado 18 abr. 2016. Disponible en http://sit.conaf.cl/.

CONAF (Corporación Nacional Forestal, CL). 2016c. Oficina de Informaciones, Reclamos y Sugerencias. Consultado 24 jun. 2016. Disponible en http://oirs.conaf.cl/.

CONAF (Corporación Nacional Forestal, CL), Ministerio de Agricultura. 2016. Política Forestal 2015-2035. Consultado 12 sep. 2016. Disponible en http://www.conaf.cl/wpcontent/files mf/1462549405politicaforestal201520351. pdfwww.conaf.cl/wp-content/files_mf/1462549405politica forestal201520351.pdf.

Congalton RG. 1991. A review of assessing the accuracy of classifications of remotely sensed data. Remote Sensing of Environment 37(1):35-46. DOI:10.1016/0034-4257(91)90048-b

Corona P. 2016. Consolidating new paradigms in large-scale monitoring and assessment of forest ecosystems. Environmen- tal Research 144:8-14. DOI: 10.1016/j.envres.2015.10.017

FAO (Food and Agriculture Organization of the United Nations, IT). 2010. Evaluación de los recursos forestales mundiales 2010, informe nacional. Roma, Italia. 68 p.

FAO (Food and Agriculture Organization of the United Nations, IT). 2014a. Comité Forestal. Directrices voluntarias sobre monitoreo forestal nacional, $22^{\circ}$ período de sesiones. Roma, Italia. Consultado 9 ene. 2016. Disponible en http:// www.fao.org/documents/card/es/c/52b0ce4e-fbb6-46d2$8 \mathrm{~d} 3 \mathrm{c}-3 \mathrm{~d} 4479711 \mathrm{e} 66 /$

FAO (Food and Agriculture Organization of the United Nations, IT). 2014b. Global forest resource assessment 2015, country report Chile. Roma, Italia. 99 p.

FAO (Food and Agriculture Organization of the United Nations, IT). 2015. Global Forest Resources Assessment. Roma, Italia. $245 \mathrm{p}$.

Giri Ch. 2012. Remote sensing of land use and land cover: principles and applications. Boca Raton, USA. Taylor \& Francis Group. 413 p.

Heilmayr R, C Echeverría, R Fuentes, E Lambin. 2016. A plantation-dominated forest transition in Chile. Applied Geography 75:71-82. DOI: 10.1016/j.apgeog.2016.07.014

Herold M, M Skutsch. 2011. Monitoring, reporting and verification for national REDD + programmes: two proposals. Environmental Research Letters 6(1):014002. DOI: 10.1111/j.1755-263X.2010.00159.x

Holmgren P, LG Marklund, M Saket, ML Wilkie. 2007. Forest monitoring and assessment for climate change reporting: partnerships, capacity building and delivery. Forest Resources Assessment Working Paper 142. Roma, Italia. FAO. 44 p.

Keenan RJ, GA Reams, F Achard, JV de Freitas, A Graiger, E Lindquist. 2015. Dynamics of Global Forest Area: Results from the FAO Global Forest Resources Assessment 2015. Forest Ecology and Management 352:9-20. DOI: 10.1016/j.foreco.2015.06.014

MacDicken KG. 2015. Global Forest Resources Assessment 2015: What, Why and How?. Forest Ecology and Management 352:3-8. DOI: 10.1016/j.foreco.2015.06.014

Miranda A, A Altamirano, L Cayuela, A Lara. 2017. Native Forest Loss in the Chilean Biodiversity Hotspot: Revealing the Evidence. Regional Environmental Change 17:285297. DOI: $10.1007 / \mathrm{s} 10113-016-1010-7$

Pelletier J, SJ Goetz. 2015. Baseline Data on Forest Loss and Associated Uncertainty: Advances in National Forest Monitoring. Environmental Research Letters 10(2):021001. DOI: 10.1088/1748-9326/10/2/021001

Romijn E, C Lantican, H Martin, E Lindquist, R Ochieng, A Wijaya, D Murdiyarso, L Verchot. 2015. Assessing Change in National Forest Monitoring Capacities of 99 Tropical Countries. Forest Ecology and Management 352:109-23. DOI:10.1016/j.foreco.2015.06.003

UACh (Universidad Austral de Chile, CL), UFRO (Universidad de La Frontera, CL), CONAF (Corporación Nacional Forestal, CL). 2014. Monitoreo de cambios, corrección cartográfica y actualización del catastro de los recursos vegetacionales nativos de la región de La Araucanía. Valdivia, Chile. UACh-CONAF. 38 p. 
